\title{
Astronomy for a Better World: IAU OAD Task Force-1 Programs for Advancing Astronomy Education and Research in Universities in Developing Countries
}

\author{
Edward Guinan $^{1}$ and Katrien Kolenberg ${ }^{2}$ \\ ${ }^{1}$ Villanova University, USA; \\ ${ }^{2}$ Harvard Smithsonian Center for Astrophysics, USA \& University of Leuven, Belgium; \\ email: edward.guinan@villanova.edu
}

\begin{abstract}
We discuss the IAU Commission 46 and Office for Astronomy Development (OAD) programs that support advancing Astronomy education and research primarily in universities in developing countries. The bulk of these operational activities will be coordinated through the OAD's newly installed Task Force 1. We outline current (and future) IAU/OAD Task Force-1 programs that promote the development of University-level Astronomy at both undergraduate and graduate levels. Among current programs discussed are the past and future expanded activities of the International School for Young Astronomers (ISYA) and the Teaching Astronomy for Development (TAD) programs. The primary role of the ISYA program is the organization of a three week School for students for typically M.Sc. and Ph.D students. The ISYA is a very successful program that will now be offered more frequently through the generous support of the Kavli Foundation. The IAU/TAD program provides aid and resources for the development of teaching, education and research in Astronomy. The TAD program is dedicated to assist countries that have little or no astronomical activity, but that wish to develop or enhance Astronomy education. Over the last ten years, the ISYA and TAD programs have supported programs in Africa, Asia, Central America and the Caribbean, the Middle East, South East and West Asia, and South America. Several examples are given.

Several new programs being considered by OAD Task Force-1 are also discussed. Other possible programs being considered are the introduction of modular Astronomy courses into the university curricula (or improve present courses) as well as providing access to "remote learning" courses and Virtual Astronomy labs in developing countries. Another possible new program would support visits of astronomers from technically advanced countries to spend their sabbatical leaves teaching and advising University Astronomy programs in developing countries. Suggestions for new Task Force -1 programs are also welcomed. Useful information about the participation of IAU members and volunteers in these programs will be discussed and practical information will be provided.
\end{abstract}

\section{Introduction}

An IAU/OAD Task Force (Task Force 1, TF-1) has been established for the development of Astronomy Education and Research to promote Astronomy teaching and research activities at universities in developing countries. This program builds on the long history of related work within the IAU's Commission 46 Program Groups. Astronomy serves to stimulate research and teaching in related STEM (Science, Technology, Engineering and Mathematics) areas and to develop the programs in regions where there is little or no Astronomy. There is also potential for developing research in the historical and cultural aspects of Astronomy which may prove important for igniting interests in 
Astronomy (and in sciences in general) in communities where there is no strong interest in the sciences. The broad appeal of Astronomy across the world makes such a program very attractive to people from diverse cultural and education backgrounds.

\section{International School of Young Astronomers (ISYA)}

The IAU ISYA program was created in 1967 and has organized 34 schools thus far. It is now hosted by OAD TF-1. The ISYA program provides concentrated expert instruction and training in basic and special topics of modern astronomy to a number of selected young astronomers or physicists with or without a graduate degree who otherwise would not have such opportunities. The IAU, in collaboration with the Norwegian Academy of Sciences and Letters, pays for the transport of faculty and participants; the host country pays for stay of faculty and participants. Currently there is one ISYA event held each year. Chair: Dr. Jean-Pierre De Greve (Belgium); Vice Chair: Kam-Ching Leung (USA)

\section{Education and Research Programs}

Several examples of programs supported by TF-1 are listed below. A few of these programs were previously hosted under the IAU C-46 Teaching Astronomy for Development and World Wide Astronomy Programs. Most of them are new programs.

(1) Visiting astronomer programs or exchange programs. (2) National or regional astronomy schools and workshops. (3) Astronomy education /research equipment and laboratory small grant program (4) Sabbatical leave visit program: grants to spend sabbaticals at a university (or institute) in a developing country to provide educational and technical advice. (5) Technology internships, e.g., instrument specialists to visit universities in developing countries. (6) Grants for the development and implementation of undergraduate astronomy courses and labs in developing countries (7) University twinning programs develop long term (at least 2 years) links and cooperative programs between institutes and universities with those in developing host countries. (8) Innovative university astronomy education and research programs not listed above (e.g., support or enhance the development of innovative teaching, laboratory and undergraduate research programs). Fresh ideas for high impact programs are most welcome.

\section{Proposal Selection Criteria}

Proposals can be submitted to the IAU/OAD for IAU financial support for education and research programs. Selection criteria and more informaiton are provided on the IAU/OAD Website http://www.astro4dev.org 